Communications in Contemporary Mathematics

Vol. 11, No. 6 (2009) 1079-1081

(c) World Scientific Publishing Company

\title{
Author Index Volume 11 (2009)
}

Adimurthi \& Santra, S.,
Generalized Hardy-
Rellich inequalities in
critical dimension and its
applications

Anguelova, I. I. \& Bergvelt, M. J., $H_{D}$-quantum vertex algebras and bicharacters

11 (2009) 367

Bartolo, R. \& Germinario, A., Convexity conditions on the boundary of a stationary spacetime and applications

Bentrad, A. \& Kichenassamy, S., Hypergeometric functions and singular solutions of wave equations with Lorentzinvariant potential

Bergvelt, M. J., see Anguelova, I. I.

Biswas, I. \& Muñoz, V., Torelli theorem for moduli spaces of $\mathrm{SL}(r, \mathbb{C})$ connections on a compact Riemann surface

Carrião, P. C., de Figueiredo, D. G. \& Miyagaki, O. H., Quasilinear elliptic equations of the Henontype: Existence of nonradial solutions

Catanese, F., Canonical symplectic structures and deformations of algebraic surfaces

Chang, K. C., The spectrum of the 1-Laplace operator

Chapouly, M., Global controllability of a nonlinear Korteweg-de Vries equation
11 (2009) 447

11 (2009) 937

11 (2009) 481

11 (2009) 739

11 (2009) 1

11 (2009) 783

11 (2009) 865

11 (2009) 495
Charro, F. \& Peral, I., Zero order perturbations to fully nonlinear equations: Comparison, existence and uniqueness

Cheng, Q.-M., Ichikawa, T. \& Mametsuka, S., Inequalities for eigenvalues of Laplacian with any order

Cho, Y. \& Ozawa, T. Sobolev inequalities with symmetry

Chu, W. \& Jia, C., Abel's method on summation by parts for elliptic hypergeometric series

de Figueiredo, D. G., see Carrião, P. C.

11 (2009) 131

11 (2009) 639

11 (2009) 355

11 (2009) 337

11 (2009) 783

Deng, Y., Jin, L. \& Peng, S., Positive solutions for elliptic equations related to the Caffarelli-KohnNirenberg inequalities do Ó, J. M., Moameni, A. \& Severo, U., Semi-classical states for quasilinear Schrödinger equations arising in plasma physics

Fang, D. \& Zhong, S., $L^{2}$-concentration phenomenon for Zakharov system below energy norm

11 (2009) 27

Fialowski, A. \& Majumdar, A., Miniversal deformations of dialgebras

11 (2009) 413

Fialowski, A. \& Penkava, M., Extensions of (super) Lie algebras

11 (2009) 709
11 (2009) 185

11 (2009) 547

. Jossi, J. D. \& Sabina de Lis, J. C., Existence and uniqueness 
of positive solutions to elliptic problems with sublinear mixed boundary conditions

Gazzini, M. \& Musina, R., Hardy-Sobolev-Maz'ya inequalities: Symmetry and breaking symmetry of extremal functions

Germinario, A., see Bartolo, R.

Gustafson, S., Nakanishi, K. \& Tsai, T.-P., Scattering theory for the GrossPitaevskii equation in three dimensions

Hagge, T. J. \& Hong, S.-M., Some non-braided fusion categories of rank three

Hayashi, N. \& Naumkin, P. I., Scattering operator for nonlinear KleinGordon equations

He, Y., see Liu, W.

Her, H.-L., Floer homology for symplectomorphism

Hong, S.-M., see Hagge, T. J.

Ichikawa, T., see Cheng, Q.-M.

Jia, C., see Chu, W.

Jin, L., see Deng, Y.

Kamran, N., Olver, P. \& Tenenblat, K., Local symplectic invariants for curves

Karel, M. \& Li, H., Some quantum vertex algebras of ZamolodchikovFaddeev type

Kichenassamy, S., see Bentrad, A.

Labarca, R., Pumariño, A. \& Rodríguez, J. A., On the boundary of topological chaos for the MilnorThurston world

Li, H., see Karel, M.

Li, S. \& Zhu, M., A sharp inequality and its applications

Liu, W. \& He, Y. Finite-dimensional special odd Hamiltonian
11 (2009) 657

11 (2009) 165

11 (2009) 585

11 (2009) 993

11 (2009) 739

11 (2009) 615

11 (2009) 771

11 (2009) 523

11 (2009) 895

11 (2009) 615

11 (2009) 639

11 (2009) 337

11 (2009) 185

11 (2009) 829

11 (2009) 447

11 (2009) 1049

11 (2009) 829

11 (2009) 433 superalgebras in prime characteristic

11 (2009) 523

Lu, G. \& Wang, M., Infinitely many even periodic solutions of Lagrangian systems on any Riemannian tori with even potential in time

11 (2009) 309

Majumdar, A., see Fialowski, A.

11 (2009) 413

Mametsuka, S., see Cheng, Q.-M.

11 (2009) 639

Micu, S., Ortega, J. H. \& Pazoto, A. F., On the controllability of a coupled system of two Korteweg-de Vries equations

11 (2009) 799

Miyagaki, O. H., see Carrião, P. C.

Moameni, A., see do Ó, J. M.

11 (2009) 783

11 (2009) 547

Moser, R., GinzburgLandau vortex lines and the elastica functional

11 (2009) 71

Muñoz, V., see Biswas, I.

Musina, R., see Gazzini, M.

Nakanishi, K., see Gustafson, S.

Naumkin, P. I., see Hayashi, N.

11 (2009) 1

11 (2009) 993

11 (2009) 657

11 (2009) 771

Navarro-Pascual, J. C. \& Sánchez-Lirola, M. G., A vector-valued version of Russo-Dye theorem

11 (2009) 1035

Oertel, U. \& Świątkowski, J., Contact structures, $\sigma$ confoliations and contaminations in 3-manifolds

11 (2009) 201

11 (2009) 165

Olver, P., see Kamran, N.

Ortega, J. H., see Micu, S.

Ozawa, T. \& Sasaki, H., Inequalities associated with dilations

11 (2009) 265

Ozawa, T., see Cho, Y.

11 (2009) 355

Pazoto, A. F., see Micu, S. 11 (2009) 799

Peng, S., see Deng, Y.

11 (2009) 185

Penkava, $\quad$ M., see Fialowski, A.

11 (2009) 709

Peral, I., see Charro, F. 11 (2009) 131

Pumariño, A., see Labarca, R.

11 (2009) 1049

Rodríguez, J. A., see Labarca, R.

11 (2009) 1049 
Roselli, P. \& Willem, M., Least energy nodal solutions of the BrezisNirenberg problem in dimension $N=5$

11 (2009) 59

Rossi, J. D., see GarcíaMelián, J.

11 (2009) 585

Ruan, W.-D., Zhang, Y. \& Zhang, Z., Bounding sectional curvature along the Kähler-Ricci flow

11 (2009) 1067

Sabina de Lis, J. C., see García-Melián, J.

11 (2009) 585

Sánchez-Lirola, M. G., see Navarro-Pascual, J. C.

Santra, S., see Adimurthi

Sasaki, H., see Ozawa, T.

Severo, U., see do Ó, J. M.

Sigalotti, L., Asymptotic analysis of periodicallyperforated nonlinear media at the critical exponent

11 (2009) 1009

Solci, M., Homogenization of energies defined on pairs set-function

11 (2009) 1035

11 (2009) 367

11 (2009) 265

11 (2009) 547

11 (2009) 459
Świątkowski, J., Oertel, U.

Tenenblat, K. Kamran, N.

Tsai, T.-P., K.

see

11 (2009) 201

Gustafson, S.

11 (2009) 165

Wang, M., see Lu, G.

Wang, S., Gerbes, holonomy forms and real structures

11 (2009) 109

Willem, M., see Roselli, P. 11 (2009) 59

Yang, Z. \& Yi, F., Valuation of European installment put option: Variational inequality approach

Yi, F., see Yang, Z.

11 (2009) 279

11 (2009) 279

Zhang, L., Asymptotic behavior of blowup solutions for elliptic equations with exponential nonlinearity and singular data 11 (2009) 395

Zhang, Y., see Ruan, W.-D. 11 (2009) 1067

Zhang, Z., see Ruan, W.-D. 11 (2009) 1067

Zhong, S., see Fang, D. 11 (2009) 27

Zhu, M., see Li, S.

11 (2009) 433 